\title{
A System Design for Facilitating Human Resource Decisions in Small and Medium Sized Enterprises in Turkey
}

\author{
S.Çağrı Şengül ${ }^{1}$, A.Orçun Sakarya ${ }^{2, *}$, H.Volkan Kaya ${ }^{3}$, Z.Ezgi Kahraman ${ }^{4}$ \\ ${ }^{1}$ Cisco Systems, Turkey \\ ${ }^{2}$ Department of Management, Faculty of Economics and Administrative Sciences, Cankaya University, Turkey \\ ${ }^{3}$ Turktrust, Turkey \\ ${ }^{4}$ Department of City and Regional Planning, Faculty of Architecture, Cankaya University, Turkey
}

Copyright $\odot 2015$ by authors, all rights reserved. Authors agree that this article remains permanently open access under the terms of the Creative Commons Attribution License 4.0 International License.

\begin{abstract}
Currently, to keep the competitive edge, firms focus on the improvement of the decision making and use different management information systems to leverage the process. In this study, it has been designed an information system which aims to support the management of human resources in the small and medium sized enterprises in Turkey. To do this, a group of human resource key point indicators have been determined for a system design which includes Mysql- and Java-based Hibernate Criteria Api, search algorithm, relational database, JSF(java server faces) Framework and Primefaces structures as methodology. It has been demonstrated by sample user interface snapshots and analysis of the system benefits that the system would serve decision makers in a number of areas including training, performance, efficiency, workforce and working time supported by its user friendly characteristics such as; real time analysis, decision making opportunities and flexibility of accessibility it offers to small and medium sized enterprises.
\end{abstract}

Keywords Human Resources, System, Decision, Key Point Indicator

\section{Introduction}

Human resource management (HRM) processes include a number of phases in an organization. These are basically personnel and time management, time measurements, payrolls, compensation, and workforce planning. Some of these phases are basically undertaken among daily activities such as record keeping and status tracking whereas, some include more "traditional" activities such as planning, recruitment, selection, orientation and training, and performance management.
In all phases, information systems (IS) are used as a facilitator to improve the quality of decisions to be made, to produce real time reports, to collect and transform data to information later to be used for employee benefit. IS is also used in different domains including operational issues (e.g. payrolls), managerial activities (e.g. promotion measurement and needs analysis), and finally in-house collaboration technologies. Still considering decision support systems; different examples can be observed in literature such as the one for the mould industry [1], pharmaceutical industry [2], apparel manufacturing [3], chemical process industry [4], and in textile industry [5]. Likewise, IS contributes a number of issues such as interactive decision making, employee control, improvement of operational processing, document sharing and reporting, and automation of the daily operations. In this case, these systems can briefly be called as human resource information systems (HRIS).

In Turkey, Small and Medium Sized Enterprises (SMEs) are mainly using IS in certain domains involving accounting, customer relations, inventory management, automation and communication [6]. When the IS usage within organizations is considered in general terms, limited access to qualified human resource required for IS operations is stated as one of the disadvantages, [7] automation of the business processes is considered as a significant potential for the SME development [8]. Accordingly, it can be envisaged that certain SMEs also lack efficient HRIS implementations in terms of issues including training, performance, efficiency, workforce and working time. HRIS, as derivative systems, can also be assumed to contribute on organizational competitiveness in this context.

Regarding the fact that the majority of the organizations in Turkey are SMEs, the goal of this study is designing a robust IS serving for HRM purposes of which especially SMEs will take the advantage.. The study is composed of four parts. Following the introduction and related literature search, the 
system design section includes the selection and reformulation rationale of a group of human resource key point indicators including training, performance, efficiency, workforce and working time to fit the required output content. Discussion part of the study focuses on the output simulation by demonstrating sample user interface snapshots and analysis of the system benefits for human resource decision makers. In the conclusion part, potential implementations for further system development areas, such as customer relations management, and brand management are also mentioned. The findings of the study will be used for the research's next phase in which a more detailed HRIS is aimed to be designed

\section{Literature Review}

Based on the literature search, the studies concerning SMEs and HRIS systems can be considered under two groups: the first group of studies generally focuses on HRIS implementations in different sized firms, whereas, the second group of studies is mostly concentrated on IS usage in SMEs which cover in brief.

Regarding the first group of studies, which is mainly composed to HRIS related researches, HRIS in which has been approached by Hendrickson [9], in general terms, where he underlines the importance the integration of traditional human resources (HR) functions with contemporary information technology (IT) competences and how these systems are expected to influence HR functions depending on structural, size and functional characteristics. HRIS adoption and different factors such as technological, organizational and environmental factors that affect the successful adoption of HRIS are discussed by Chakraborty [10] in their study. They concluded that organizational size, management commitment are significant factors in this context. At the same context, Jawahar and Harindran [11] have suggested a research model which involves judgmental components and made related assumptions.

$\mathrm{HR} / \mathrm{IT}$ relation in the organizational context is also one of the important topics that came into being in the literature. $\mathrm{Ji}$ et al. [12] have investigated the effect of organizational identity on sustainable performance of HRM in IT. They concluded that collectivist orientation of organizational identity should moderate the HRM and execution of the sustainable strategy effects positively firms' performance in environmental protection. They have also concluded that HRM affects positively the performance in sustainable development. In this regard, they have also offered practitioners guidelines concerning the role of organizational identity for HRM in IT to contribute to firms' sustainable development. At the same framework, Tansley and Watson [13] have focused the effect of organizational context in HR systems development in the framework of strategic exchange in an organizational contextual setting. They deduced that the negotiations between HRIS implementation project group members and the people they contact, promote a cultural learning which is then reflected in corporate level.

Regarding the impact of HRIS implementations, Weeks [14] concentrated in the HRIS' impact on different aspects of HR process such as training, reassignment, retention, job performance, satisfaction, undertaking of job related-tasks, productivity and absenteeism. Accordingly, it has been deduced that organization efficiency and effectiveness can be empowered by HRIS implementation which are expected to prevail both in scale and scope in the future. Hussain [15] focused on the impact of HRIS on HR professionals in their study. They have reached the conclusion that strategic use of HRIS is augmenting without regard to company size and add value to $\mathrm{HR}$ operations. Another important finding in the study states that SMEs are implementing HRIS more promptly due to increased need for legislation of accountability and equal opportunities monitoring. They have also concluded that although the costs of similar systems might be perceived as high, the return on investment is increasing in SMEs. In their research, Nagendra [16] investigated the range of HRIS usage in mid to large sized organizations and advantages retrieved of human resource planning sub functions. According to their findings, mostly accepted HRIS aspect is the identification of unfilled job positions. They have also added that IS and HR strategy alignment serves HR planning efficiency through HRIS skills inventory. In this way, more intelligent capabilities should also be offered by HRIS in order to increase HR planning effectiveness.

Additionally covered literature also includes alternative topics in which HRIS is focused in different dimensions. For example, Dery [17] mainly focusing on the implementation of a HRIS system, investigated the formation of actor networks. In the framework of Actor Network Theory, they underlined the impacts of sophisticated technology usage and the role of new actor network in providing HR services and by observing translation process in a different way. Obeidat [18] has concentrated on the diffusion of innovation via HRIS in the pharmaceutical sector. The researcher deduced that innovation diffusion and HRIS functions are related with each other. It has also been added that the compatibility of innovation with the social system within an organization the more likely that the HRIS would be adopted. Finally, Zafar [19] focused to information security issues of the HRIS in his study and made suggestions in a wider array including, data evaluation, security policies, legislative and regulatory policies, security architecture, system integration procedures, training and related programs and risk analysis. He also suggested a research model for solving security issues.

Second group of studies mainly concentrates on the implementation IS in different organizational sizes, some concentrating on SMEs including; interorganizational dimensions of ISs' impact on SMEs [20], IS architecture in SMEs [21], IS competencies in SMEs [22], IS systems strategy in SMEs [23] and SME IT-IS classification dimension [24]. Interesting findings of those researches indicate the multifunctionality and significance of the HRIS 
along with various IT and IS in different organizational sizes. Hence, it can also be intuited that potential results that might be deduced from its practical implementations may also contribute on the literature.

In this regard, based on the literature covered; this study, which is mostly practical mainly stands in between two groups of studies stated in the previous paragraph.

\section{System Design: System Metrics and Architecture}

As previously mentioned, system design mainly serves as a resource is to enable HR department managers to optimize the decision making process. To display the outputs (mostly in the graphical form) a number of key metrics act as inputs and embedded in formulas showed in the following section.

\subsection{Key Point Indicators and System Design Methodology}

System design process can be segregated into two groups. The first group mainly deals with determination of the "key point indicators (KPIs)". KPIs are related with HRM metrics being used in the system. The second group is related to the system design properties.

KPIs acting as inputs basically cover parameters related with funding issues, employee satisfaction, time usage, performance monitoring, training and lastly control and evaluation of the employees on department basis. Consolidating all these headings, KPIs follows adopted from [25] can be summarized as follows;

- Recruitment KPIs

- Training key point indicators (KPIs)

- Performance KPIs

- Working KPIs

- HR KPIs

- Workforce KPIs

The second group, in other words, "system design properties" involves the software selection. KPIs are mainly reflected via Graphical User Interface (GUI) structure to the clients, whereas Java Server Faces (JSF) and Primafaces are used for the completion of the user-friendly interfaces. Inclusion of the Java also serves platform independence as the system can easily be integrated into different operating systems in this way. Data for the parameters used within the system are stored in a database which has been established by using MySql. An example for the related database is "common employee" database which mainly contains data on employee identity, department code and per person salary. Another database called "performance database" contains data on year, department code, the number of interviewed people, and department threshold mainly used on measuring KPIs where a company budget allocation case is estimated. In other words, "thresholds" are quotients used for the distribution of the budget wherever required. Therefore, it can also be observed that certain KPIs such as training, performance, working time and sales are measured on departmental basis, whereas the remaining KPIs are company-wide. Finally, it should be added that data entry for the parameters covered below is entered by at least one authorized person where the number can be increased according to the varying organizational scales or structures. In the study, it has been assumed that certain parameters are entered into the system arbitrarily for simulation purposes. Below, KPIs measurement is explained in more details.

\section{Recruitment KPIs}

These metrics basically provide information on recruitment costs and various recruitments statistics of company according to years. Accordingly, they enable managers to observe the value results of statistics of previous years and make decisions on the operation of the recruitment processes needed in order to increase number of employment applicants or reducing the recruitment costs. In this context, reputation or profit improvements can be done by adjusting recruitment expenses to get more visibility between applicants. Furthermore, acknowledgement of the recruitment statistics may also permit the manager reducing the recruitment cost by making some adjustments in interview time, and related expenses on per hour cost basis.

Under this section, various arbitrary parameters such as open position number, related year, total number of $\mathrm{CV}$ for open position, number of submitted $\mathrm{CV}$ for interview process, interview time for an applicant, other expenses (advertisements, materials and background checking processes etc.) and per hour cost for an employee interview are included.

Parameter values are entered at yearly basis for proper visualization purposes for KPIs in following sections, including this one. To calculate different costs, system uses following formulas;

Employee Interview Cost $=$ value of per hour cost $\mathrm{x}$ value of interview time

Recruitment Cost $=$ Employee Interview Cost + Other Expense Cost

\section{Training KPIs}

This section's KPIs help manager decide on training cost of the department on department salary (budget allocation for training in the department), the percentage of the training cost with respect to over total cost and the number of the staff to be trained, and the number of training hours per employee in terms of the years. Manager is able to see each department's training budget ratio according to the total training budget, each department's training threshold, and the total employee salary level of the entire organization. Here KPIs also aim managers to acknowledge the percentage of each department's training budget in terms of the total cost. Moreover, manager may observe how many people are trained and the length of this training in terms of number of training hour per employee. 
As a result, these statistics demonstrate the money spent on training, the number of trained people and the duration of training process. Deduced results are expected to help manager to improve decision making during employee performance evaluation in the departments and distribution of the profits between the departments. Arbitrary parameters for this process include; year, total annual training budget, total employee salary (based on department), department training threshold, department training hours, and number of department trained people. Total cost without overtime cost which is not entered as an arbitrary data is retrieved from the employee database that is mentioned above. For this section, formulas used by the system are;

Department Training Budget $=($ Total Training Budget $\mathrm{x}$

Training Threshold) / Department Total Salary

Total Cost $=$ Recruitment Total Cost + Total Employee Salary + Total Training Budget

Department Training Budget Percent (in terms of Total Cost) $=(100 \times$ Department Training Budget $) /$ Total Cost

Department Trained People Percent $=(100 \times(\#$ of Department Trained People)) / Department Total Employee

Training Hours per Employee $=$ Department Total Training Time / Department Total Employee

\section{Performance KPIs}

This section of the KPIs covers measurement of yearly employee performance and achievement level or the pre-defined performance criteria on department basis. To do this, KPIs enable managers monitor certain issues such as completion time of the performance appraisals and cover employee performance grades according to their performance points to distinguish between low and high performing employees. The most important issue to be kept in mind here is that performance evaluation is not undertaken in a continuous basis but in specific periods and by related department rather than HR department alone.

In similar performance evaluation periods, first assumption that could be made is that performance appraisal completion time generally is identified by the management as one week. Therefore, manager can observe the weekly basis percentage of the appraisals and decide on success of the performance evaluation process accordingly. For example, if a department has 10 employees and average number of the employee performance evaluated is five per week the achievement of the performance evaluation process is fifty per cent. Considering that this ratio may vary on a department basis, manager may be able to benchmark the evaluation performance according to pre-defined target levels. Formula serving for the calculation of the percent in question is;

Department number of interviewed people percent $=$ (number of interviewed people x 100) / department total employee count
The result of this formula also serves to observe the employee performance status of the each department according at yearly basis and provides clues for future activities to improve the interview process (e.g training of the staff for holding more efficient interview sessions). Given training budget and the number of trained people from the previous section, manager can identify the performance point's threshold, this time for required training purposes. After the identification of the thresholds and comparison of the employee performance points (including knowledge, abilities, and skills to perform the tasks) with the threshold, manager is able to see the percent of employees that have high and low rate of performance.

The related arbitrary parameters then involve: Year, department name, number of interviewed employee (completed appraisals), performance points threshold (for comparing and deciding low/high performance) and performance points of the employee interviewed (for each employee grade per department). According to the number of interviewed people, managers set performance points of interviewed people. Then, they may compare the points obtained to the department performance point's thresholds they set heuristically. If employee performance points are greater than or equal to the threshold, then, they may assume that employee has a high performance; else employee has a low performance and set the profile of the required trainings (or the threshold of the training budget) for the following year for example.

\section{Working time KPIs}

Working time KPIs results show managers the total absenteeism time, total overtime in hours, total net-working time in hours and total overtime costs of departments of the company according to years. Manager can observe absenteeism time, overtime and net-working time for deciding on related department performances and can determine required promotion tools or prizing policies (e.g. distributing the bonuses according to these assessments).Working time KPIs also facilitate managers observing the overtime costs of each department in the company.

Arbitrary parameters for this section are: year worked, department name (arbitrarily set), hourly late time (absenteeism job time), hourly overtime due, per hour overtime salary and submitted work time that is decided by company management for total one year work time. Formulas used by the system for this section are as follows; Net-Working Time $=$ Submitted Work Time + Overtime - Late Time

Overtime Cost $=$ Overtime $\mathrm{x}$ per Hour Overtime Salary

According to absenteeism time, net-working time and overtime observation for each department, manager can make ad-hoc decisions on department productivity. For example, if net-working time is smaller than the submitted 
work time, a manager can investigate potential solutions in order to reach required net-working time and decide on different tools for decreasing the absenteeism time per worker. This formula may also enable manager to observe overtime costs of each department according to years for deciding on different compensation policies on department basis.

\section{Human resource efficiency KPIs}

Under this heading, KPI is mostly perceived in financial terms in order to measure efficiency. To do this, calculation of departmental basis employee profit in such a way to reflect the cost profile that is related with employment is mainly under consideration. Then, this measure can be used as an indicator for managers to identify distribution thresholds to be used for different priorities such as training and recruitment needs.

The arbitrary parameters of this section are year, sales turnover amount (total sales for one year) and department threshold (profits' distribution thresholds of departments). In order to get the profit amount, firstly the program uses the recruitment cost of the recruitment KPIs; training cost from the training KPIs section, labor cost of the company from employee total salary and finally overtime cost from working time indicators section to find the annual total cost of the whole company. Related cost formulas are shown below;

Labor Cost $=$ Total Employee salary + Total Overtime

$$
\text { Cost of Company }
$$

Total Cost $=$ Recruitment Cost + Training Cost + Labor

Cost

Total Profits $=$ Sales Turnover $($ Total Sales $)-$ Total Cost

And to calculate the profits of the each department;

Department Profit $=$ Total Profit $\mathrm{x}$ Department Threshold

Here, it should also be noted that formulas 3.11 to 3.14 mainly serve the calculation of different metrics of remaining KPIs.

\section{Workforce KPIs}

Manager can observe the company workforce, according to the results of the workforce statistics by distinguishing between full and part time employees. The results of the part and full-time employees can also be assumed as the ratio of core over contingent employees as an indirect indicator while setting business-level strategies.

Here, the manager can observe the average of work life length for each current employee of the company and each leaved employee. The rationale lies behind the fact that if length of an employee work life is lower than expected; the manager can take some action for increasing the average work life. Under this heading turnover rate is also calculated as an important indicator.

Arbitrary parameters included are; year, number of full time employees and persons left. Here the number of part-time is excluded due to the special structural features of SMEs to be able to generalize sector differences.

Turnover rate formula then becomes;

Turnover rate=Number of employees left/Number of full time employees x 100

\subsection{System Process Flow}

To reach the charts which are covered in the next section, a user (e.g. system administrator) begins with entering arbitrary values belonging to KPIs into the system which is categorized according to object-oriented logic.

In the first place, system administrator establishes database connection by inserting the password provided. In this way, password usages enable administrators to visualize all pages and make changes whenever required. Moreover, password usage also provides a rapid access and abolishes the need of making extra definitions. Then, administrator reaches the fields on web application interface in which he will be entering arbitrary data. At this stage, system administrator may also define user roles at departmental basis and transfer his authority to the selected staff in different departments, where, he may also import required data from different file formats such as MS Excel and so on. Then, system transfers data registries for every KPI to pure Java classes by using Prima Faces Framework. This data is then shaped by using object-oriented logic and imported to the related databases via Hibernate which guarantees total consistency. When a change or an update is required for registered data in the database, automatic queries on the web interface facilitate the process. In this step, system is able to display the KPI constant or arbitrary data stored in pure Java classes on meaningful charts or graphical outputs again by the help of Hibernate technology. JSF and Prima Faces applications on Web application interface enable the visualization of the charts on any location with Internet access.

\section{Discussion: System outputs and Potential Benefits}

\subsection{System Outputs}

Selected system outputs mainly involve the ones about recruitment, working time, training, performance and workforce, in other words the KPIs. Some of these KPI outputs are in departmental basis (e.g. working time, training and performance) whereas some are in related parameters and algorithms basis.

\subsubsection{Recruitment}

In recruitment section, the first group of KPIs mainly deals with recruitment statistics for which, the user is able to observe some general data including the number of $\mathrm{CVs}$ 
collected, the number of CVs included in the interview process and accordingly, the percentage of the CVs considered for the interview on the CVs collected after the job advert for example. Here, the average time spent in hours for the interview is also entered as an arbitrary data into the system. Accordingly, the manager may overview the mainstream of the job demand and performance of the candidate consideration. Outputs are shown in Figure 1.

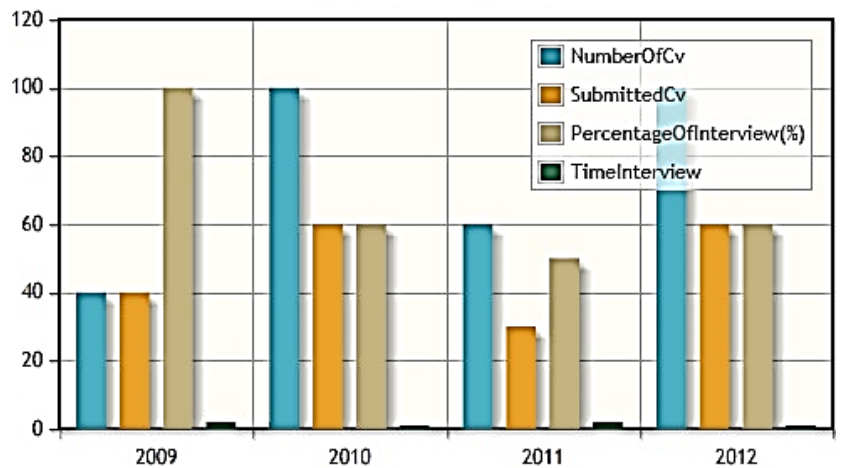

Figure 1. Recruitment statistics

In the same section, system also enables managers to monitor the cost structure of the recruitment process as a result of formulas (3.1) and (3.2). According to Figure 2, the manager is also able to observe the ratio of interview cost over total cost in monetary terms.

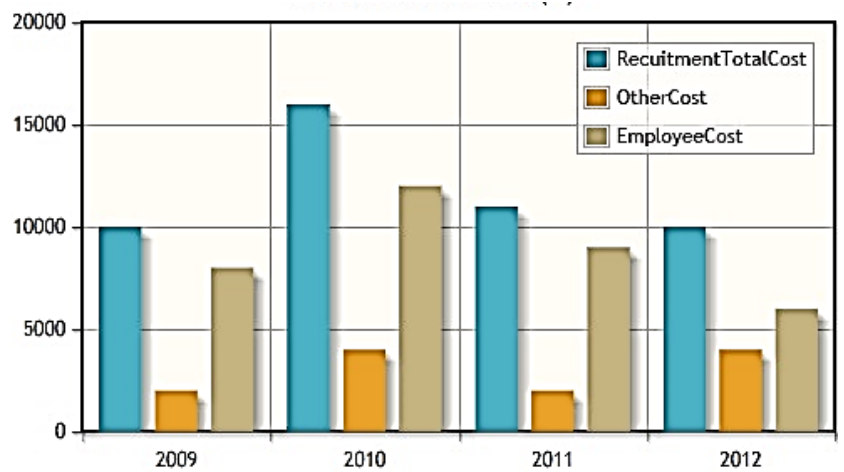

Figure 2. Recruitment cost

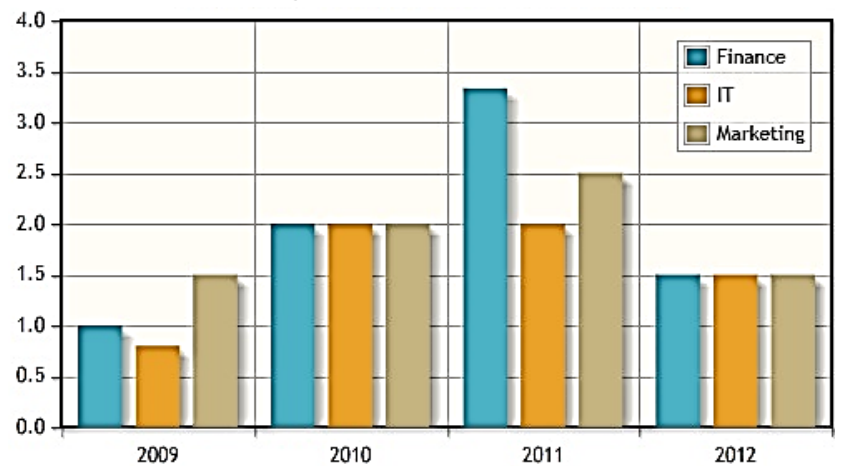

Figure 3. Ratio of training cost versus salary

\subsubsection{Training}

Under training section, cost structure of the training process in the organization is basically focused. After deducting the results of formulas 3.3 to 3.7 , system output provides the ratio of the training cost versus salary provided in department basis for the managers who need to indirectly compare the budget portion afforded for training and salary in departmental basis.

Results in Figure 3 and Figure 4 permit managers to observe priority level of training among departments in cost basis for different years and decide on the potential allocation of the training budgets according to different future strategies.

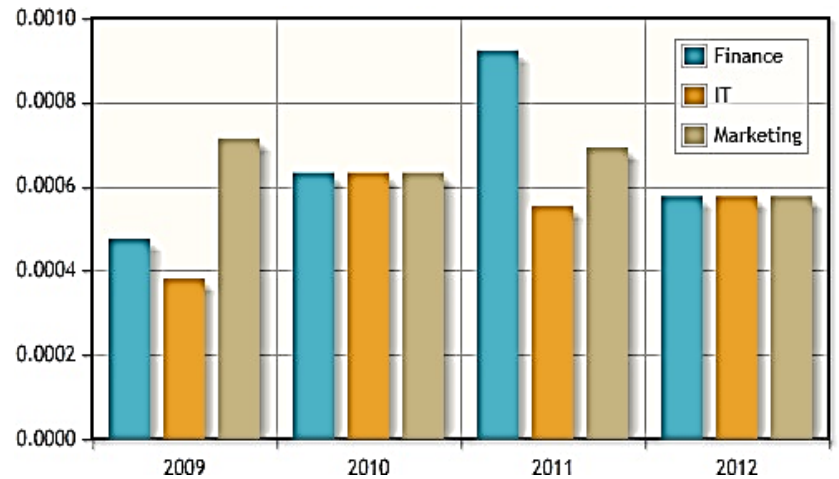

Figure 4. Percentage of the training cost over total cost

Figure 5 displays the performance results of the training budget allocated at a yearly basis for different departments. In other words, this figure shows the contribution of the training budget on the training process realized and enables managers to analyze end result of budget allocations again for future budget evaluation decisions.

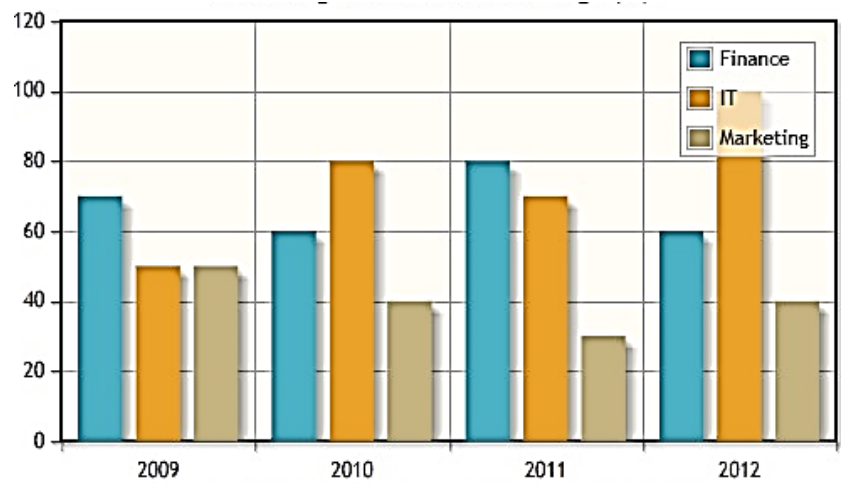

Figure 5. Percentage of the people trained

\subsubsection{Performance}

Under "performance" section, selected results in the context of appraisals and employees performing according to pre-defined criteria for different departments are provided.

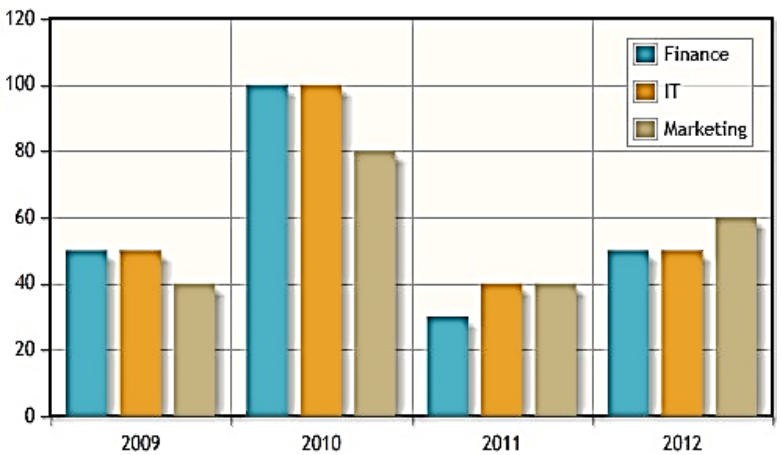

Figure 6. Percentage of the appraisals completed 
Figure 6 shows the results of the completed appraisals' for different departments. In this context, when difference in the number of department workers is omitted, this figure also displays the comparative performance of departments as a result of the appraisal process undertaken. Regarding the temporary profile of appraisal periods and the extra effort required for evaluation certain cases; department-based performance monitoring may serve staff in charge for time efficiency purposes.

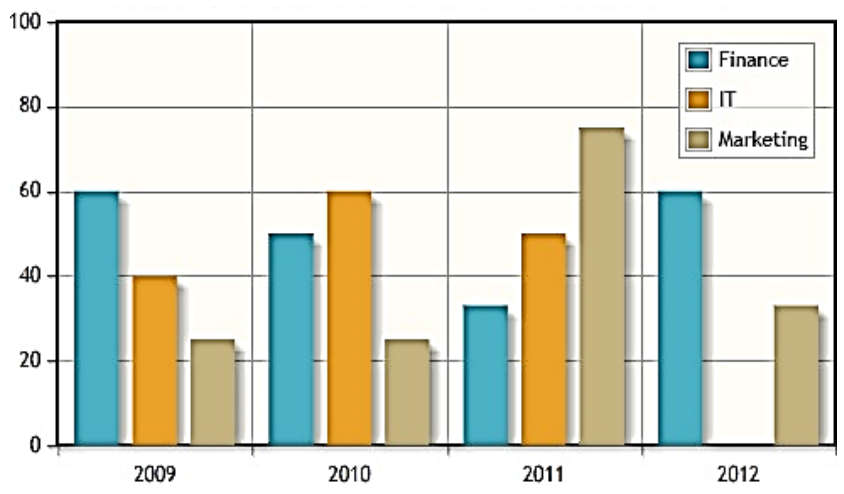

Figure 7. Percentage of the low performing employees

In conjunction with Figure 6, Figure 7 displays the percentage of the low performing employees again in department basis which might enable managers evaluating the results of the past appraisal/recruitment processes and again, formulation of the potential compensation approaches.

\subsubsection{Working time}

Results under this section mainly include measurements about working and absenteeism time and overtime cost in departmental basis. According to the results of Figure 9 and 10 , managers are able to monitor total working and absenteeism time for the years considered when they need to evaluate different departments' performances. Furthermore, these two different results also provide managers clues during the search for potential reasons of the performance deficiencies and developing tools for improving department basis attendance.

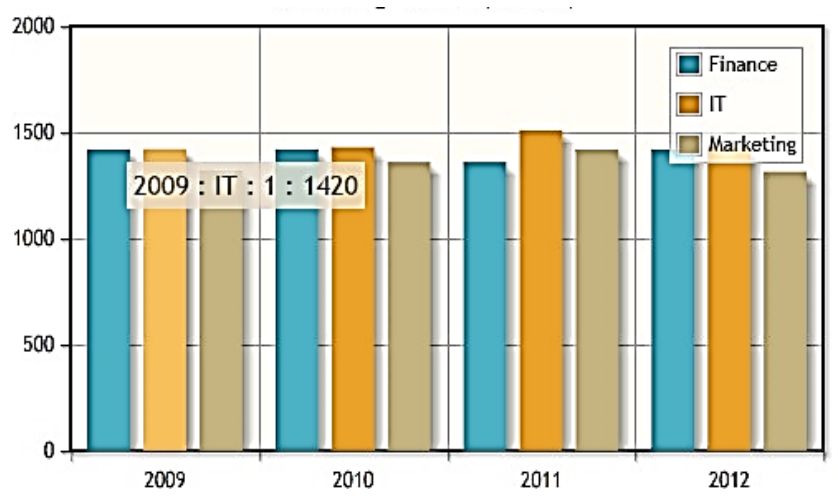

Figure 8. Working time (total hours)

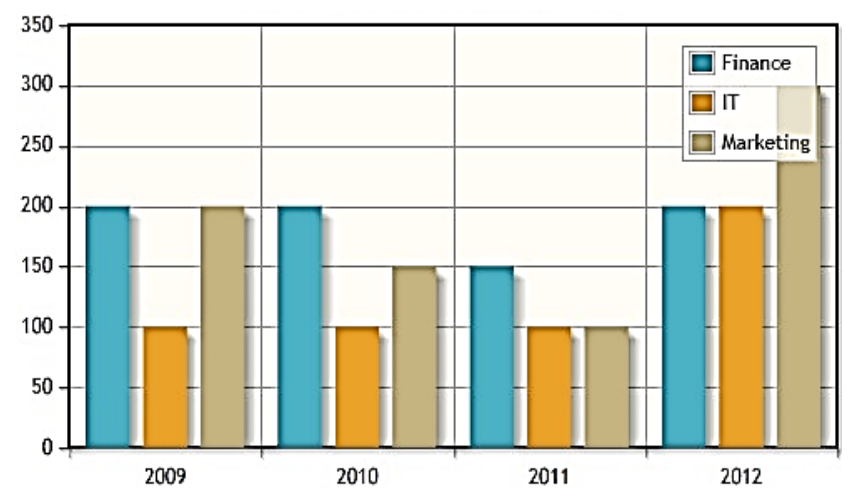

Figure 9. Absenteeism time (total hours)

Another result that can be deduced in this section is the cost of working overtime costs for the organization of different departments. When the Figure 10 is observed in connection with two previous figures, it can also be envisaged possible ties between absenteeism time and allocated resources for the implementation of the workflow in department basis, again both for performance evaluation purposes. In this context, when a department has a low level of absenteeism and low overtime cost, a manager can conclude a higher performance for the related department and vice-versa.

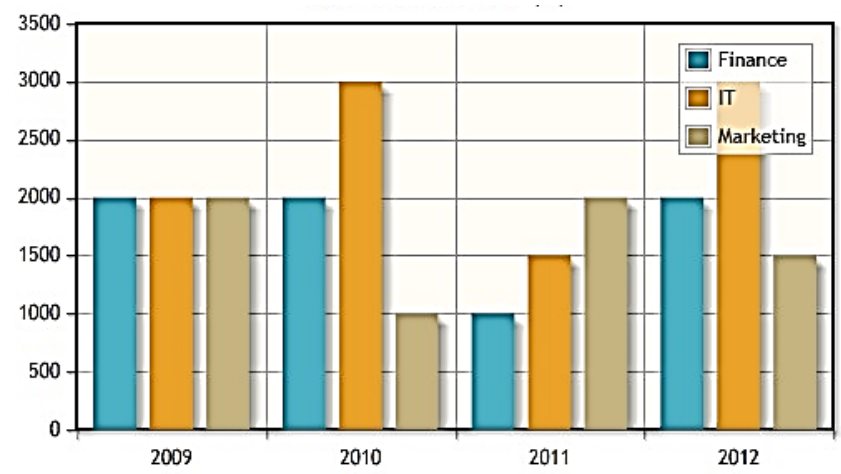

Figure 10. Working overtime cost

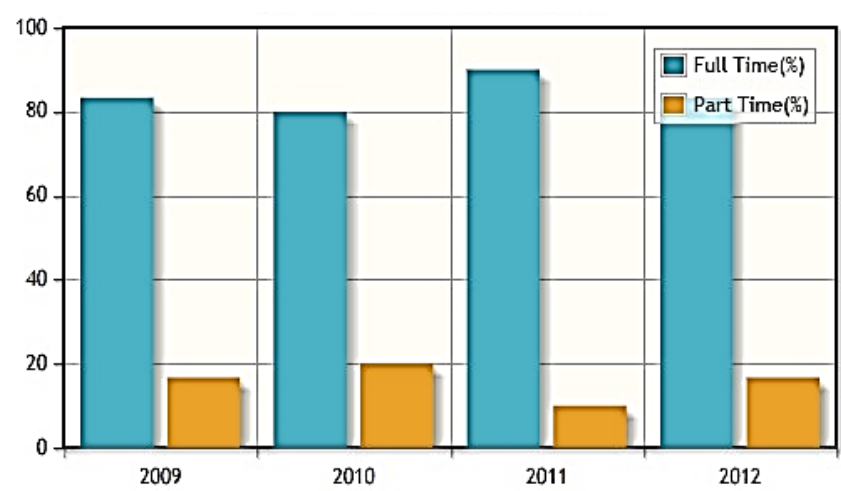

Figure 11. Total workforce dispersion

\subsubsection{Workforce}

Here, two different results that can be deduced by the system are basically considered: general dispersion of the 
workforce as full time and part time workers and the other which is related with worker satisfaction. Results of Figure 11 mainly displays the ratio of core and contingent employees in the organization and leverages managers make decisions on a number of issues such as resource allocation for benefits, modifying the function and roles that will be assigned to different types of employees and so on.

Figure 12 shows turnover rate that system retrieves as the result of the formula (3.15). According to this figure, by excluding part-time employees (which status may vary in different organizations) managers are able to monitor the ratio of the total employees who leave the organization and consider the related results for formulating policies to increase the employee loyalty and commitment.

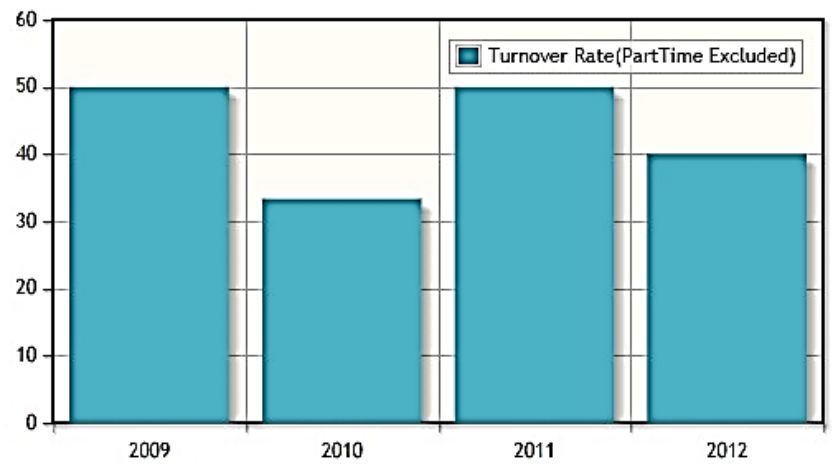

Figure 12. Turnover rate

\subsection{Potential Benefits for small and medium sized enterprises}

Benefits of the system can be summarized under different headings. Main system contributions arise at the point where it may facilitate SME managers' decision making process by providing relevant input and output combination in a way that they are queried by the manager or anybody who is authorized. System benefits can be classified as follows;

Ease of use: The system also eases the decision making process by constituting a part of the key human resources issues including recruitment, performance evaluation and other process that were previously mentioned by providing multifaceted results for different evaluation purposes. Here, it can also be added that an HR staff may not need a computer literacy at the expert level to operate the system.

Little number of operational staff: Due to the number of limited number of staff in certain SMEs, staff allocation constraints of certain SMEs for the data entry may arise. At this point, the system provides insertion flexibility, where, small amounts of data may only require only one staff for entry. This can also be estimated as a cost effective advantage for the SMEs. In other words, an SME may not need "big data" to operate the system and allocate its staff accordingly.

Real time analysis and decision making opportunities: The system provides an opportunity to managers and decision makers to make various analyses by using real-time data which can also be used for integrating different processes between recruitment, presence in the firm and performance evaluation. The system is able to make monitoring of human resource activities of the company by using real time data which enables the company to respond to the dynamic effects in both internal and external environments which may create vulnerabilities for small-scaled organizations in certain cases. Another benefit of this system is that it provides the efficiency in the employee appraisal and promotion processes by using department information in an organized way. System also provides an effective monitoring of employees in different departments and make related decisions whenever needed, all of which can be assumed as effective solutions in small enterprises when a professional control mechanism lacks.

Flexibility of accessibility and integration: Another set of contributions is related with system specifications themselves. To reach the benefits stated in the previous paragraph, the system provides customized reporting options and a common consistent database which can also be accessed online which also provides utilization flexibility regardless of location and time. The system also is integrated into various Internet and web applications already used by different types of organizations and provides fast, reliable and secure access by the help of encryption mechanisms it contains. Another specification advantage of the system is its ability to use different information sources and that multiple parties may be able to participate the decision making process by the integration of the file sharing, communication (collective e-mail, message sending) and e-signature modules into the system. In the same context, easy integration opportunities may also enable organizations to use the system with present applications in the SME such as accounting modules and so on. Such ease of integration can also be imagined as a cost-effective solution for an SME.

\section{Conclusions}

In this study, a HRIS as a preliminary level of a more detailed system design process which targets the needs of the SMEs is designed. Currently, this system has a limited number of functions which support different but mutually integrated phases of the HR process in an organization some of which are highlighted below;

Besides time and cost effective solutions offered by the system, robustness and easy integration opportunities to different modules forefront as the major advantage regarding the usage of the existing module packages in different organizations, especially the SMEs. User friendly interface is another important feature of the system which diminishes the need for deep computer literacy and potential complications during operations. Therefore, system is aimed to serve different effective HR decision making processes by providing different data retrieval options to clients and managers on a timely and quantitative manner. In this context, it can also be anticipated that system might mainly 
facilitate semi-structured HR decision making activities in SMEs.

Within the framework of this study, further studies may concentrate on for additional system development areas, including customer relations management and brand management depending on different technical requirements. On a possible pre-implementation phase, interviews with sector experts for the improvement of system functions constitute another area of further research.

\section{REFERENCES}

[1] C.Silva, L.Roque, A.Almaida. MAPP - A web-based decision support system for the mould industry, Decision Support Systems, Vol. 42, 999-1014,2006.

[2] W.Hernandez, J.M. Rivera. A Production Information System, an application to pharmaceutical industry, Computers Industrial Engineering, Vol. 33, No (1-2), 15-18, 1997.

[3] Z.X. Guo, W.K.Wong, C.Guo. A cloud-based intelligent decision-making system for order tracking and allocation in apparel manufacturing, International Journal of Production Research, Vol. 52, No. 4, 1100-1115, 2014.

[4] G.Ying, S. Zhigang, A.Kokosis. Agent-based intelligent system development for decision support in chemical process industry, Expert Systems with Applications, Vol. 36, 11099-11107, 2009.

[5] H.J. Hwang, J.Seruga. An Intelligent Supply Chain Management System to Enhance Collaboration in Textile Industry, International Journal of u- and e- Service, Science and Technology, Vol. 4, No. 4, 2011.

[6] Online Available: TUBISAD. SME Research 2010 Informatics Industry Association Online available from http://www.tubisad.org.tr/Tr/Activities/Sayfalar/edonusum.a $\operatorname{spx}$

[7] Online Available: Ministry of Science, Information and Technology, SME Strategy and Action Plan, Online available from http://www.sanayi.gov.tr/Files/Documents/K OSGEB_Katalog.pdf

[8] Online Available: Ministry of Development, Information Society Strategy and Action Plan 2015-2018 Online available fromhttp://www.kalkinma.gov.tr/Lists/EylemVeDi gerPlanlar/Attachments/25/20152018_bilgi_toplumu_strateji si_ve_eylem_plani.pdf

[9] A, Hendrickson. Human resources information systems: Backbone of contemporary human resources, Journal of Labor Research, Vol.14, No. 3, 381-394, 2003.

[10] A.R. Chakraborty, N.N.A Mansur. Adoption of Human Resource Information System: A Theoretical Analysis, Procedia - Social and Behavioral Sciences 75, 473-478, 2013.

[11] D. Jawahar, K.N Harindran. Role of Affect in the Acceptance of Human Resource Information Systems, The IUP Journal of Management Research, Vol. 7, No 2, 54-74,
2013.

[12] J. Li, G.Tang, Y.Chen. Firms' human resource in information system and sustainable performance: Does their organizational identity matter?, The International Journal of Human Resource Management, Vol. 23 No.18, 3838-3855, 2012.

[13] C.Tansley, T.Watson. Strategic exchange in the development of Human Resource Information Systems (HRIS), New Technology, Work and Employment, Vol. 15, No.2, 108-122, 2000 .

[14] K.O.Weeks. An Analysis of Human Resource Information Systems impact on Employees, Journal of Management Policy and Practice Vol.14, No 3, 35-49, 2013.

[15] Z.Hussain, J.Wallace, N.E.Cornelius. The use and impact of human resource information systems on human resource management professionals, Information \&Management, 44, 74-89, 2007.

[16] A.Naghendra, M.Desphande. Human Resource Information Systems (HRIS) in HR planning and development in mid to large sized organizations, Procedia - Social and Behavioral Sciences, Vol.133, $61-67,2014$.

[17] K.Dery, R.Hall, N.Wailes, S.Wiblen. Lost in translation? An actor-network approach to HRIS implementation, Journal of Strategic Information Systems, Vol. 22, 225-237,2013.

[18] B.Y.Obeidat. The relationship between innovation diffusion and Human Resource Information System (HRIS), Perspectives of Innovations, Economics \& Business, Vol. 12, No. 3, 41-58, 2012.

[19] H.Zafar. Human resource information systems: Information security concerns for organizations, Human Resource Management Review, 23,105-113, 2013.

[20] J.Kauremaa, M. Kärkkäinen, T. Ala-Risku. Customer initiated interorganizational information systems: The operational impacts and obstacles for small and medium sized suppliers, International Journal of Production Economics, Vol. 119, 228-239, 2009.

[21] R.Bhagwat, M.K. Sharma. Information system architecture: a framework for a cluster of small and medium-sized enterprises (SMEs), Production Planning \& Control: The Management of Operations, Vol. 18 No. 4, 283-296, 2007.

[22] P.Cragg, M.Caldaira, J. Ward. Organizational information systems competences in small and medium-sized enterprises, Information \& Management,Vol. 48, 353-363, 2011.

[23] M.Levy, P.Powell. Information systems strategy for small and medium sized enterprises: an organisational perspective, Journal of Strategic Information Systems, Vol. 9, 63-8,2000.

[24] J.L. Salmeron, S. Bueno. An information technologies and information systems industry-based classification in small and medium-sized enterprises: An institutional view, European Journal of Operational Research, Vol. 173, 10121025, 2006.

[25] Online Available: Business Performance Managament-Top 75 KPIs for Human Resource Department Online available from

http://www.businessperformancemanagement.info/2011/11/t op-75-kpis-for-human-resources-department.html 